\title{
31. Rb-Sr SYSTEMATICS OF BASALTS FROM THE HAWAIIAN-EMPEROR VOLCANIC CHAIN
}

\author{
Marvin A. Lanphere and G. Brent Dalrymple, U. S. Geological Survey, 345 Middlefield Road, \\ Menlo Park, California 94025 \\ and \\ David A. Clague, Department of Geology, Middlebury College, Middlebury, Vermont 05753
}

\begin{abstract}
Rubidium and strontium concentrations and strontium isotopic compositions have been determined for 70 samples of basalt from 22 volcanoes in the Hawaiian Islands-Emperor Seamount volcanic chain. The mean ${ }^{87} \mathrm{Sr} /{ }^{86} \mathrm{Sr}$ ratios for basalts of the tholeiitic suite range from 0.70316 to 0.70411 and have a mean value and standard deviation of $0.70369 \pm 0.00028$. Basalts of the alkalic suite show a range from 0.70290 to 0.70394 and have a mean value and standard deviation of $0.70344 \pm 0.00026$. In addition to the difference between these two suites, the variations in $\mathrm{Sr}$ isotopic composition within each suite indicate real differences in the $\mathrm{Rb} / \mathrm{Sr}$ and ${ }^{87} \mathrm{Sr} /{ }^{86} \mathrm{Sr}$ ratios of the source regions from which individual flows were derived.

The isotopic composition of $\mathrm{Sr}$ in tholeiitic basalts erupted during the past 43 m.y., from Kilauea Volcano on Hawaii to the Hawaiian-Emperor bend, is relatively uniform; but ${ }^{87} \mathrm{Sr} /{ }^{86} \mathrm{Sr}$ ratios decrease systematically from the Hawaiian-Emperor bend to 65-m.y.-old Suiko Seamount. This pattern may be related to the difference in age between the underlying crust and the volcano.

There is essentially no correlation between ${ }^{87} \mathrm{Sr} /{ }^{86} \mathrm{Sr}$ and $\mathrm{Rb} / \mathrm{Sr}$ for basalts of the Hawaiian-Emperor chain. These data do not support the concept of a mantle isochron indicating a time when the source region for the basalts was established.

The source regions for basalts of the Hawaiian-Emperor chain probably lie within the lithospheric plate; the Hawaiian hot spot or melting anomaly is probably an energy source that produced melting in the overlying plate. Variations in $\mathrm{Sr}$ isotopic composition within individual volcanoes and between volcanoes indicate that the source regions for basalt are quite heterogeneous in detail.
\end{abstract}

\section{INTRODUCTION}

Studies of the volcanic rocks of the Hawaiian Islands over the last 50 years have provided a wealth of data that have greatly influenced current concepts of volcanism and of crust and mantle evolution. The Hawaiian Islands also have figured prominently in development of certain aspects of plate tectonic theory, particularly the hot-spot hypothesis. According to this hypothesis, linear volcanic island chains, such as the Hawaiian Islands-Emperor Seamount volcanic chain (Figure 1), are produced as a crustal plate moves relative to a source of magma in the asthenosphere. A volcano forms above the hot spot, is eventually moved away from the magma source, and a new volcano is formed on the sea floor behind it. The hypothesis was first proposed by Wilson $(1963 a, b)$ to explain the origin of the Hawaiian Islands and other northwest-trending island chains in the Pacific Ocean. He suggested that the source of magma is in the stable core of a convection cell, and that the source is moving horizontally more slowly than the near surface part of the cell. Christofferson (1968) subsequently coined the term "hot spot," which he visualized as a stationary magma source at the top of an ascending convection current in the mantle. He suggested that volcanic islands were produced by magmatic pulsations from the hot spot. Morgan (1972a, b) further developed the hypothesis by suggesting that hot spots are the surface manifestations of deep mantle plumes that are fixed in location relative to each other and provide the driving force for sea-floor spreading. According to Morgan, the hot spots are the source of material erupted as volcanic rocks.

The corollary that the ages of volcanoes in a linear island chain increase progressively from one end to the other has been tested extensively in the Hawaiian-Emperor Chain by a number of geochronologic studies. Twenty-seven individual volcanoes are now dated; the 


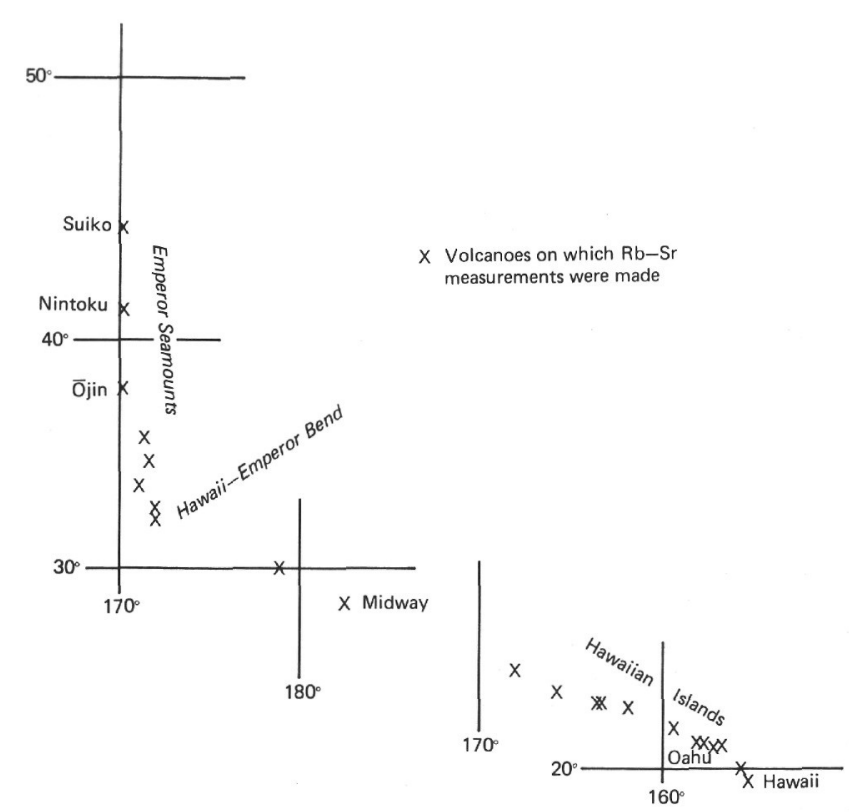

Figure 1. Generalized map of Hawaiian Islands-Emperor Seamount chain, showing locations of 22 volcanoes for which $R b$-Sr measurements were made in this study. A more detailed location map is given in Jackson et al. (this volume).

results are consistent with an average volcanic propagation velocity of about $8 \mathrm{~cm} /$ year, from the active volcano of Kilauea on the island of Hawaii to 65-m.y.-old Suiko Seamount in the Emperor Chain (Dalrymple et al., this volume). In addition, paleomagnetic studies of basalt drill core from Midway Atoll (Grommé and Vine, 1972), Suiko Seamount (Kono, this volume), and Meiji Guyot (Marshall, 1978) indicate that the Hawaiian hot spot has been in the general vicinity of its present latitude for the past 65 m.y., and that the volcanoes have been carried northwestward by motion of the Pacific plate relative to the hot spot.

$\mathrm{Rb}-\mathrm{Sr}$ systematics provide a different type of test of the Hawaiian hot spot. If, as Morgan suggests, the source of the magma is a deep mantle plume, then we would expect the chemistry of the derivative lavas to be relatively constant through time, or at least to evolve in a systematic way. The isotopic composition of $\mathrm{Sr}$ in basalts from the Hawaiian-Emperor Chain is a sensitive indicator of the chemical constancy of the source region for the basalts that form the Hawaiian-Emperor volcanoes. The principal objectives of this study were to use $\mathrm{Sr}$ isotope relationships as a tracer in determining (1) whether there is a pattern in the $\mathrm{Sr}$ isotopic compositions that documents either homogeneity or change in the source region for Hawaiian volcanic rocks during the past 65 m.y., and (2) whether there is a difference in the isotopic composition of $\mathrm{Sr}$ in tholeiites and alkalic basalts from the same volcano that might yield information about whether the source regions for the two basalt types are the same or different.

\section{PREVIOUS Rb-Sr STUDIES OF HAWAIIAN VOLCANIC ROCKS}

Several Hawaiian volcanic rocks were included in early investigations, most of which were for purposes of reconnaissance, where radiogenic $\mathrm{Sr}$ was used as an isotopic tracer to study geologic processes. No detailed study of the $\mathrm{Sr}$ isotope systematics of Hawaiian volcanic rocks has been undertaken; published data include samples from only 8 of the 15 volcanoes that comprise the main Hawaiian Islands.

Approximately $110 \mathrm{Sr}$ isotopic analyses of Hawaiian volcanic rocks have been reported previously. A number of these analyses were made by different investigators on the same samples. Most of the data from rocks of the tholeiitic and alkalic suites from the main shield volcanoes are compiled in Table 1. Results for a few samples that cannot be related to an individual volcano have been omitted. Data for the nephelinic basalts of the Honolulu Volcanic Series on Oahu are not included, because these rocks are not part of the main shield-building stage of the volcanoes. Nearly all of the approximately $30 \mathrm{Sr}$ isotopic measurements on rocks of the Honolulu Volcanic Series were made in detailed studies by Powell and DeLong (1966) and Lanphere and Dalrymple (in press).

The data in Table 1 have been adjusted to a ${ }^{87} \mathrm{Sr} /{ }^{86} \mathrm{Sr}$ value of 0.7080 for the Eimer and $\mathrm{Amend} \mathrm{SrCO}_{3}$ standard, except for those from two studies where this standard was not analyzed. Because most data were collected several years ago, the analytical precision is not up to present standards. Most of the ${ }^{87} \mathrm{Sr} / 86 \mathrm{Sr}$ ratios for basalts from seven volcanoes in the main Hawaiian Islands lie between 0.703 and 0.705 , with a few (5) values lower than 0.703 and a few (3) higher than 0.705 (Figure 2). These data do not show any sytematic difference in Sr-isotopic composition between basalts of the tholeiitic and alkalic suites. As a whole, the data suggest an average ${ }^{87} \mathrm{Sr} /{ }^{86} \mathrm{Sr}$ ratio of about $0.704 \pm$ 0.001 for basalts from the main Hawaiian Islands.

\section{SAMPLES STUDIED}

Seventy samples of basalt from 22 volcanoes in the Hawaiian-Emperor chain were analyzed in this study (Table 2). Locations of these volcanoes are shown schematically in Figure 1; a more detailed index map of the Hawaiian-Emperor chain is given in Jackson et al. (this volume). Macdonald and Katsura (1964) classified the basalts erupted from volcanoes on the main Hawaiian islands into three groups which, in order of eruption are (1) a tholeiitic suite that forms the bulk $(>99 \%)$ of the shield volcanoes, (2) an alkalic suite that forms thin caps on the shields shortly after caldera collapse, and (3) a nephelinic suite that is erupted from satellite vents after a prolonged period ( 2 to 5 m.y.) of quiescence and erosion. Basalt samples recovered from the western Hawaiian Ridge and the Emperor Seamounts are similar chemically and petrologically to those in the main Hawaiian Islands (Clague, 1974; Clague et al., 1975; 
TABLE 1

Previous Sr Isotopic Measurements on Basalts from the Hawaiian Islands ${ }^{\mathrm{a}}$

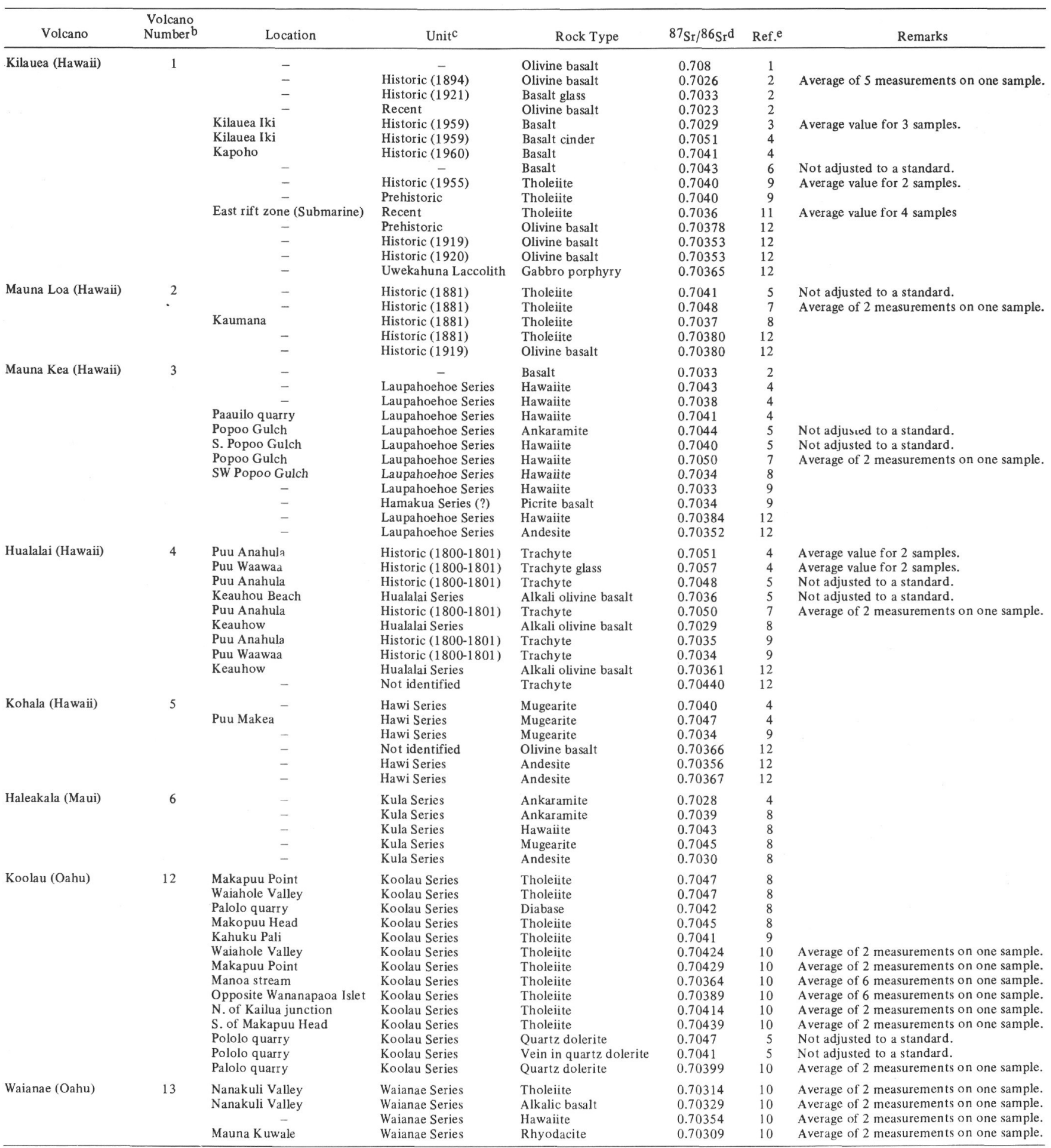

aResults for Honolulu Volcanic Series, Oahu not included. See Powell and DeLong (1966) and Lanphere and Dalrymple (in press).

bFrom Barger and Jackson (1974).

cSamples assigned to map series where possible.

d $86 \mathrm{Sr} /{ }^{88} \mathrm{Sr}$ ratios normalized to a value of $0.1194 .{ }^{87} \mathrm{Sr} / 86 \mathrm{Sr}$ ratios adjusted to a value of 0.7080 for Eimer and $\mathrm{Amend}^{\mathrm{SrCO}} 3 \mathrm{standard}$, except where noted.

eReferences: 1 - Gast (1960) 7 - Powell et al. (1965)

2 - Faure and Hurley (1963)

3 - Hedge and Walthall (1963)

4 - Lessing and Catanzaro (1964)

5 - Hamilton (1965)

7 - Powell et al. (196
8 - Bence (1966)

9 - Hedge (1966)

10 - Powell and DeLong (1966)

6 - Heier et al. $(1965)$

12 - O'Nions et al. (1977) 


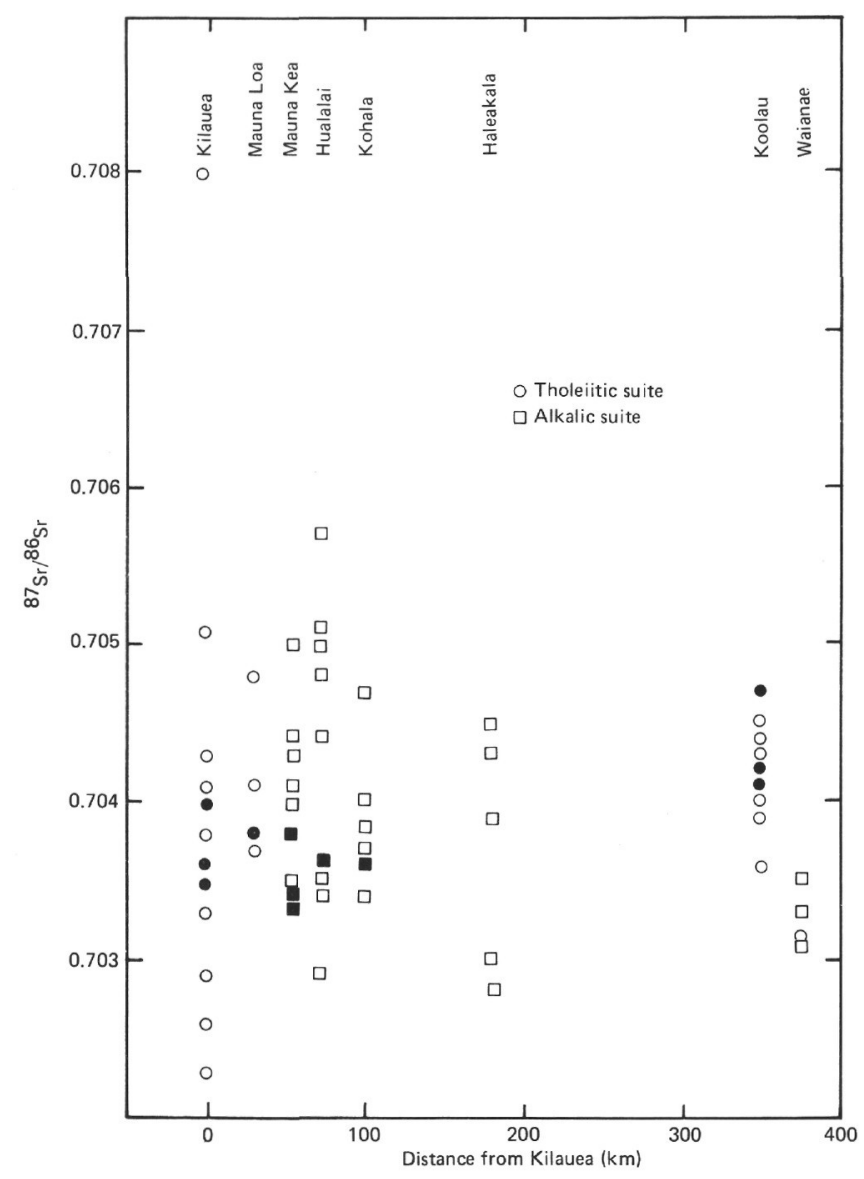

Figure 2. Previously published ${ }^{87} \mathrm{Sr} /{ }^{86} \mathrm{Sr}$ ratios plotted against distance from Kilauea Volcano measured along trend of Hawaiian Islands. Rocks of the nephelinic suite are not included. Filled symbols indicate there are two or more samples at that datum.

Clague et al., this volume). The basalt samples in this study include 37 from the tholeiitic suite, 28 from the alkalic suite, and 5 from the nephelinic suite. Both tholeiitic and alkalic basalts were analyzed from 8 volcanoes. Twenty-eight of the samples are from outcrop, 22 are from dredge hauls, and 20 are from drill cores. Three of the latter group are from Midway Atoll (Ladd et al., 1967, 1970); the other 17 are from sites drilled during DSDP Leg 55.

\section{ANALYTICAL TECHNIQUES}

The size of samples varied considerably. For samples from DSDP Leg 55, approximately $70 \mathrm{~g}$ of basalt was powdered and a split of about $5 \mathrm{~g}$ used for $\mathrm{K}_{2} \mathrm{O}, \mathrm{Rb}$, and Sr measurements. For the HIG, TSDY, and A7 suites, $\mathrm{Rb}$ and $\mathrm{Sr}$ measurements were made on aliquants, weighting several grams, that were split from powdered material used for other chemical analyses. For the remaining samples, $25 \mathrm{~g}$ or more of basalt were powdered and used for $\mathrm{Rb}$ and $\mathrm{Sr}$ measurements. The samples of basalt were pulverized to less than $0.074 \mathrm{~mm}$. $\mathrm{Rb}$ concentrations were measured by X-ray fluorescence or atomic absorption spectrophotometry; $\mathrm{Sr}$ concentrations were measured by either X-ray fluorescence, atomic absorption spectrophotometry, or isotope dilution. For Rb measurements, the standard deviation of analytical precision is estimated to be about \pm 6.8 per cent for samples analyzed by W. P. Doering (Doering, 1968), \pm 3 per cent for those analyzed by D. A. Clague (Clague, 1974), and $\pm 2 \mathrm{ppm}$ if $\mathrm{Rb}<15 \mathrm{ppm}$ and $3 \mathrm{ppm}$ if $\mathrm{Rb}>15 \mathrm{ppm}$ for samples analyzed by J. Morris. For $\mathrm{Sr}$ measurements, the standard deviation of analytical precision is estimated to be about \pm 6.5 . per cent for samples analyzed by W. P. Doering using X-ray fluorescence (Doering, 1968), \pm 3 per cent for samples analyzed by D. A. Clague using atomic absorption spectrophotometry (Clague, 1974), and \pm 1 per cent for samples analyzed by A. L. Berry using isotope dilution.

The isotopic composition of $\mathrm{Sr}$ was measured on unspiked aliquants of the powdered basalt. Mass spectrometry was done with an automated $30.48-\mathrm{cm}$-radius, $90^{\circ}$-sector, single-collector instrument utilizing a triplefilament source and digital data acquisition.

The measured ${ }^{86} \mathrm{Sr} /{ }^{88} \mathrm{Sr}$ ratios were normalized to a value of 0.1194 to determine the instrumental discrimination. Half this discrimination correction was applied to the measured ${ }^{87} \mathrm{Sr} / 86 \mathrm{Sr}$ ratios to obtain normalized ratios. The normalized ${ }^{87} \mathrm{Sr} /{ }^{86} \mathrm{Sr}$ ratios were then adjusted to a value of 0.71014 for the $\mathrm{NBS} 987 \mathrm{SrCO}_{3}$ standard, which is the value obtained on the same mass spectrometers which yield a value of 0.7080 for the older Eimer and $\mathrm{Amend}_{\mathrm{SrCO}_{3}}$ standard. Twenty analyses of the NBS 987 standard made during the course of this study yielded a mean ${ }^{87} \mathrm{Sr} /{ }^{86} \mathrm{Sr}$ ratio of 0.71023 with a standard deviation of 0.00011 and a standard deviation of the mean of 0.00002 . Each $\mathrm{Sr}$ composition data set for a basalt sample consisted of 12 mass scans which then were averaged and corrected for discrimination. An individual experiment generally consisted of 2 to 4 data sets (that is, $n=2$ to 4 ). The ${ }^{87} \mathrm{Sr} /{ }^{86} \mathrm{Sr}$ ratio for an experiment (Table 2) is the mean of the values for individual data sets, and the error is the square root of the sum of the squares of the standard deviations of individual data sets divided by the number of data sets. For example, the basic data for Sample 430A-4-2, 110-118 cm, from Ōjin Seamount, are as follows:

$\begin{array}{cc}\text { Data Set } & { }^{87} \mathrm{Sr} / 86 \mathrm{Sr} \\ 1 & 0.70346 \pm 28 \\ 2 & 0.70357 \pm 14 \\ 3 & 0.70356 \pm 19\end{array}$

For this analysis, $n$ is 3 and the mean ${ }^{87} \mathrm{Sr} / 86 \mathrm{Sr}$ ratio and standard deviation are $0.70353 \pm 12$.

Replicate measurements were made on 9 samples; each of these analyses represents a separate dissolution of powdered basalt. The total spread in the ${ }^{87} \mathrm{Sr} / 86 \mathrm{Sr}$ ratio for these replicate measurements ranged from 0.00001 to 0.00036 , and for 7 of the 9 samples was less than 0.00013 . The ${ }^{87} \mathrm{Sr} / 86 \mathrm{Sr}$ ratios for these 9 samples are weighted mean values calculated by weighting individual measurements by the inverse of their estimated variances. Probably the best estimate of precision is the pooled estimate of standard deviation which, for the 9 samples having replicate measurements, is 0.015 per cent or \pm 0.00011 in the ${ }^{87} \mathrm{Sr} / 86 \mathrm{Sr}$ ratio, the same value obtained for replicate measurements of the NBS 987 
standard. Replicate measurements generally were made if the initial measurement had a large analytical uncertainty or if the strontium isotopic composition was substantially different from that of other basalts from the same volcano. Thus, the population of replicate analyses is not an ideal one for establishing an estimate of precision, and precision may be somewhat better. A more realistic estimate of precision probably is the pooled estimate of standard deviation of the 7 samples having a difference in ${ }^{87} \mathrm{Sr} / 86 \mathrm{Sr}$ ratio of less than 0.00013 . The pooled estimate of standard deviation for these 7 samples is 0.007 per cent or \pm 0.00005 in the ${ }^{87} \mathrm{Sr} / 86 \mathrm{Sr}$ ratio.

Age corrections, based on the $\mathrm{K}-\mathrm{Ar}$ age of each volcano (Dalrymple et al., this volume) were applied to the adjusted ${ }^{87} \mathrm{Sr} /{ }^{86} \mathrm{Sr}$ ratios of the three samples having $\mathrm{Rb} / \mathrm{Sr}$ ratios greater than 0.1 .

\section{RESULTS}

The range in rubidium and strontium concentrations and $\mathrm{Rb} / \mathrm{Sr}$ ratios for the three volcanic suites (Table 2) are as follows:

$\begin{array}{llll} & \mathrm{Rb}(\mathrm{ppm}) & \mathrm{Sr}(\mathrm{ppm}) & \mathrm{Rb} / \mathrm{Sr} \\ \text { Tholeiitic Suite } & 0-19.1 & 206-484 & 0-0.048 \\ \text { Alkalic Suite } & 4.4-95 & 380-1681 & 0.010-0.062 \\ \text { Nephelinic Suite } & 8-95 & 75-640 & 0.031-0.148\end{array}$

Sample A7-43-33, a nepheline phonolite of the alkalic eruptive stage from Kōkō Seamount was not included. ${ }^{87} \mathrm{Sr} /{ }^{86} \mathrm{Sr}$ ratios for the tholeiitic suite fall in the range $0.70316 \pm 10$ to $0.70411 \pm 8$, for the alkalic suite, $0.70290 \pm 15$ to $0.70394 \pm 9$, and for the nephelinic suite, $0.70351 \pm 19$ to $0.70443 \pm 22$. The mean value and standard deviation for the 37 tholeiites are 0.70369 \pm 0.00028 , and for the 27 alkalic basalts are $0.70344 \pm$ 0.00026 .

\section{DISCUSSION}

Evaluation of the $\mathrm{Sr}$ isotopic composition data can be done at several different levels to get at the objectives listed earlier. These levels include (1) variation within a single suite, either tholeiitic or alkalic, from an individual volcano, (2) differences between the tholeiitic and alkalic suites from an individual volcano, (3) variation within either the tholeiitic or alkalic suites along the entire Hawaiian-Emperor chain, and (4) differences between the alkalic and tholeiitic suites along the chain. The sample populations for the first two levels, in particular, are quite small, but some inferences can be drawn.

Three or more basalts from a single suite were analyzed for six different volcanoes. The standard deviation of the ${ }^{87} \mathrm{Sr} / 86 \mathrm{Sr}$ ratios ranges from 0.00003 to 0.00044 . The standard deviations of 8 tholeiites from Koolau Volcano, 4 alkalic basalts from Kōkō Seamount (volcano no. 74), and 5 tholeiites from Suiko Seamount are greater than 0.00010 , and for these three volcanoes it appears that the variations in ${ }^{87} \mathrm{Sr} /{ }^{86} \mathrm{Sr}$ ratios reflect small heterogeneities in the source region beneath the volcanoes.

For the entire group of basalts from the Hawaiian-Emperor chain there is a small but significant (at the $99 \%$ confidence level) difference in mean strontium composition between the tholeiites and the alkalic basalts. Histograms of these results are shown in Figure 3. It is obvious from Figure 3 that the strontium isotopic compositions of the two basalt suites do not form normal distributions. However, the means of the samples will have an approximately normal distribution according to the central limit theorem. Thus, differences in means can be tested using standard statistical methods. Derivation of the two basalt types from different source regions in the mantle is a simple way to interpret the differences in mean strontium compositions of the two suites. This conclusion may not be valid, however, because it entails pooling data for volcanic rocks derived over a period of $65 \mathrm{~m} . \mathrm{y}$. from a source or sources as yet poorly defined. These ambiguities are removed by separately considering the data for tholeiites and alkalic basalts from each volcano.

The ${ }^{87} \mathrm{Sr} / 86 \mathrm{Sr}$ ratios for alkalic basalts from two volcanoes, Kohala and Waianae in the main Hawaiian Islands, an unnamed seamount (volcano no. 20) west of Nihoa Island, and Suiko Seamount, are lower than the ratios for tholeiites from the same volcano. There is no difference in ${ }^{87} \mathrm{Sr} /{ }^{86} \mathrm{Sr}$ in the two rock suites from Midway Island and Kōkō Seamount (volcano no. 74). Alkalic basalts from Daikakuji and Ōjin Seamounts have higher ${ }^{87} \mathrm{Sr} /{ }^{86} \mathrm{Sr}$ ratios than tholeiites. The mean ${ }^{87} \mathrm{Sr} /{ }^{86} \mathrm{Sr}$ ratios and standard deviations for these eight volcanoes, with the number of rock samples in parentheses, are as follows:

$$
\text { Tholeiitic Suite Alkalic Suite }
$$

$\begin{array}{lll}\begin{array}{l}\text { Kohala } \\ \text { Volcano }\end{array} & 0.70380 \pm 6(2) & 0.70344 \pm 12(2) \\ \begin{array}{l}\text { Waianae } \\ \text { Volcano }\end{array} & 0.70388 \pm 10(2) & 0.70375 \pm 6(2) \\ \begin{array}{l}\text { Volcano } \\ \text { No. } 20\end{array} & 0.70407 \pm 13(1) & 0.70332 \pm 17(1) \\ \begin{array}{l}\text { Midway } \\ \text { Island }\end{array} & 0.70375 \pm 18(1) & 0.70372 \pm 6(2) \\ \begin{array}{l}\text { Daikakuji } \\ \text { Seamount }\end{array} & 0.70375 \pm 14(1) & 0.70390 \pm 4(2) \\ \begin{array}{l}\text { Kōko Sea- } \\ \text { mount } \\ \text { (volcano }\end{array} & 0.70319 \pm 22(1) & 0.70327 \pm 23(3) \\ \text { no. } 74) & & \\ \begin{array}{l}\text { Ojin Sea- } \\ \text { mount }\end{array} & 0.70333 \pm 28(1) & 0.70349 \pm 4(4) \\ \begin{array}{l}\text { Suiko Sea- } \\ \text { mount }\end{array} & 0.70324 \pm 7(5) & 0.70313 \pm 15(4)\end{array}$

The number of samples from any single volcano is too small to determine with certainty whether the small isotopic differences reflect differences in the source regions of the two basalt types. The $\mathrm{Sr}$ isotopic data, as a whole, are consistent with the hypothesis that both tholeiitic and alkalic basalt liquids of a single volcano are derived from a source region having approximately the same $\mathrm{Rb} / \mathrm{Sr}$ ratio and $\mathrm{Sr}$ isotopic composition. As discussed below, however, the $\mathrm{Sr}$ isotopic composition of the source region for the Emperor Seamounts is different from that of the Hawaiian Ridge. Tatsumoto (1978) concluded that $\mathrm{Pb}$ isotopic variations in tholeiitic 
TABLE 2

$\mathrm{Rb}$ and $\mathrm{Sr}$ Concentrations and Sr Isotopic Compositions for Basalts from the Hawaiian-Emperor Chain

\begin{tabular}{|c|c|c|c|c|c|c|c|c|c|c|}
\hline $\begin{array}{l}\text { Sample } \\
\text { No. }\end{array}$ & $\begin{array}{l}\text { Volcano or } \\
\text { Seamount }\end{array}$ & $\begin{array}{l}\text { Volcano } \\
\text { No.a }\end{array}$ & Lat. (N) & Long. & Unit & Rock Type ${ }^{b}$ & $\begin{array}{c}\mathrm{Rb}^{\mathrm{c}} \\
(\mathrm{ppm})\end{array}$ & $\begin{array}{c}\mathrm{Sr} c \\
(\mathrm{ppm})\end{array}$ & $\mathrm{Rb} / \mathrm{Sr}$ & ${ }^{87} \mathrm{Sr} /{ }^{86} \mathrm{Sr}^{\mathrm{d}}$ \\
\hline $\begin{array}{l}\text { 1D515-1 } \\
8 \times 002-1\end{array}$ & $\begin{array}{l}\text { Kilauea (Hawaii) } \\
\text { Kilauea (Hawaii) }\end{array}$ & $\begin{array}{l}1 \\
1\end{array}$ & $\begin{array}{l}19^{\circ} 28.8^{\prime} \\
19^{\circ} 25.2^{\prime}\end{array}$ & $\begin{array}{l}154^{\circ} 54.0^{\prime} \mathrm{W} \\
155^{\circ} 18.0^{\prime} \mathrm{W}\end{array}$ & $\begin{array}{l}\text { Historic (1790) } \\
\text { Puna Series }\end{array}$ & $\begin{array}{l}\text { Tholeiite (O) } \\
\text { Tholeiite (O) }\end{array}$ & $\begin{array}{l}8.1 \\
7.5\end{array}$ & $\begin{array}{l}327 \\
346\end{array}$ & $\begin{array}{l}0.025 \\
0.022\end{array}$ & $\begin{array}{l}0.70379 \pm 7 \\
0.70348 \pm 11 \\
0.70348 \pm 43 \\
0.70345 \pm 28 \\
0.70348 \pm 17\end{array}$ \\
\hline $\begin{array}{l}\text { 1D615-3 } \\
\text { 1D659-3 }\end{array}$ & $\begin{array}{l}\text { Kohala (Hawaii) } \\
\text { Kohala (Hawaii) }\end{array}$ & $\begin{array}{l}5 \\
5\end{array}$ & $\begin{array}{l}20^{\circ} 07.2^{\prime} \\
20^{\circ} 07.2^{\prime}\end{array}$ & $\begin{array}{l}155^{\circ} 35.4^{\prime} \mathrm{W} \\
155^{\circ} 35.4^{\prime} \mathrm{W}\end{array}$ & $\begin{array}{l}\text { Polulu Series } \\
\text { Polulu Series }\end{array}$ & $\begin{array}{l}\text { Tholeiite (O) } \\
\text { Tholeiite (O) }\end{array}$ & $\begin{array}{l}1.4 \\
1.0\end{array}$ & $\begin{array}{l}262 \\
206\end{array}$ & $\begin{array}{l}0.005 \\
0.005\end{array}$ & $\begin{array}{l}0.70374 \pm 13 \\
0.70377 \pm 16 \\
0.70390 \pm 11 \\
0.70386 \pm 10\end{array}$ \\
\hline $\begin{array}{l}\text { 72M036 } \\
\text { 72M048 } \\
\text { 71WAIK-8F } \\
\text { 71WAIK-10F } \\
\text { 71WMOL-1 }\end{array}$ & $\begin{array}{l}\text { Kohala (Hawaii) } \\
\text { Kohala (Hawaii) } \\
\text { East Molokai } \\
\text { East Molokai } \\
\text { West Molokai }\end{array}$ & $\begin{array}{r}5 \\
5 \\
10 \\
10 \\
11\end{array}$ & $\begin{array}{l}20^{\circ} 04.7^{\prime} \\
20^{\circ} 08.0^{\prime} \\
21^{\circ} 10.9^{\prime} \\
21^{\circ} 10.9^{\prime} \\
21^{\circ} 09.1^{\prime}\end{array}$ & $\begin{array}{l}155^{\circ} 46.0^{\prime} \mathrm{W} \\
155^{\circ} 33.4^{\prime} \mathrm{W} \\
156^{\circ} 56.1^{\prime} \mathrm{W} \\
156^{\circ} 56.9^{\prime} \mathrm{W} \\
156^{\circ} 58.0^{\prime} \mathrm{W}\end{array}$ & $\begin{array}{c}\text { Hawi Series } \\
\text { Hawi Series } \\
- \\
- \\
-\end{array}$ & $\begin{array}{l}\text { Alkalic basalt }(\mathrm{O}) \\
\text { Alkalic basalt }(\mathrm{O}) \\
\text { Tholeiite }(\mathrm{O}) \\
\text { Tholeiite }(\mathrm{O}) \\
\text { Tholeiite }(\mathrm{O})\end{array}$ & $\begin{array}{r}48.2 \\
4.4 \\
6.4 \\
6.8 \\
19.1\end{array}$ & $\begin{array}{r}1681 \\
437 \\
339 \\
330 \\
402\end{array}$ & $\begin{array}{l}0.029 \\
0.010 \\
0.019 \\
0.021 \\
0.048\end{array}$ & $\begin{array}{l}0.70357 \pm 11 \\
0.70332 \pm 34 \\
0.70372 \pm 9 \\
0.70365 \pm 18 \\
0.70411 \pm 8\end{array}$ \\
\hline $\begin{array}{l}71 \text { WMOL-3 } \\
5 \times 767-1 \\
5 \times 108-2 \\
5 \times 323-1 \\
5 \times 301-0\end{array}$ & $\begin{array}{l}\text { West Molokai } \\
\text { Koolau (Oahu) } \\
\text { Koolau (Oahu) } \\
\text { Koolau (Oahu) } \\
\text { Koolau (Oahu) }\end{array}$ & $\begin{array}{l}11 \\
12 \\
12 \\
12 \\
12\end{array}$ & $\begin{array}{l}21^{\circ} 09.6^{\prime} \\
21^{\circ} 18.6^{\prime} \\
21^{\circ} 18.6^{\prime} \\
21^{\circ} 22.8^{\prime} \\
21^{\circ} 22.8^{\prime}\end{array}$ & $\begin{array}{l}157^{\circ} 14.0^{\prime} \mathrm{W} \\
157^{\circ} 39.4^{\prime} \mathrm{W} \\
157^{\circ} 39.4^{\prime} \mathrm{W} \\
157^{\circ} 54.6^{\prime} \mathrm{W} \\
157^{\circ} 54.6^{\prime} \mathrm{W}\end{array}$ & $\begin{array}{l}\text { Koolau Series } \\
\text { Koolau Series } \\
\text { Koolau Series } \\
\text { Koolau Series }\end{array}$ & $\begin{array}{l}\text { Tholeiite (O) } \\
\text { Tholeiite (O) } \\
\text { Th leiite (O) } \\
\text { Tholeiite (O) } \\
\text { Tholeiite (O) }\end{array}$ & $\begin{array}{l}8.3 \\
0 \\
0.7 \\
0.4 \\
1.8\end{array}$ & $\begin{array}{l}396 \\
376 \\
351 \\
451 \\
437\end{array}$ & $\begin{array}{l}0.021 \\
0 \\
0.002 \\
0.001 \\
0.004\end{array}$ & $\begin{array}{l}0.70409 \pm 23 \\
0.70368 \pm 26 \\
0.70407 \pm 12 \\
0.70406 \pm 12 \\
0.70389 \pm 14\end{array}$ \\
\hline 65MAKI-1 & Koolau (Oahu) & 12 & $21^{\circ} 18.9^{\prime}$ & $157^{\circ} 50.0^{\prime} \mathrm{W}$ & Koolau Series & Tholeiite (O) & 1.5 & 418 & 0.004 & $\begin{array}{l}0.70362 \pm 24 \\
0.70358 \pm 18 \\
0.70359 \pm 15\end{array}$ \\
\hline $65 \mathrm{~PB}-2$ & Koolau (Oahu) & 12 & $21^{\circ} 19.1^{\prime}$ & $157^{\circ} 50.7^{\prime} \mathrm{W}$ & Koolau Series & Tholeiite $(\mathrm{O})$ & 0.2 & 461 & 0.0004 & $\begin{array}{l}0.70376 \pm 17 \\
0.70365 \pm 14 \\
0.70369 \pm 11\end{array}$ \\
\hline $\begin{array}{l}\text { 65SAL-26 } \\
\text { 65KAPAA-4 } \\
\text { 5X667-3 }\end{array}$ & $\begin{array}{l}\text { Koolau (Oahu) } \\
\text { Koolau (Oahu) } \\
\text { Waianae (Oahu) }\end{array}$ & $\begin{array}{l}12 \\
12 \\
13\end{array}$ & $\begin{array}{l}21^{\circ} 22.0^{\prime} \\
21^{\circ} 23.0^{\prime} \\
21^{\circ} 24.0^{\prime}\end{array}$ & $\begin{array}{l}158^{\circ} 54.1^{\prime} \mathrm{W} \\
158^{\circ} 46.6^{\prime} \mathrm{W} \\
158^{\circ} 07.2^{\prime} \mathrm{W}\end{array}$ & $\begin{array}{l}\text { Koolau Series } \\
\text { Koolau Series } \\
\text { Waianae Series }\end{array}$ & $\begin{array}{l}\text { Tholeiite (O) } \\
\text { Tholeiite (O) } \\
\text { Tholeiite (O) }\end{array}$ & $\begin{array}{r}6.3 \\
15.9 \\
2.2\end{array}$ & $\begin{array}{l}484 \\
443 \\
411\end{array}$ & $\begin{array}{l}0.013 \\
0.036 \\
0.005\end{array}$ & $\begin{array}{l}0.70412 \pm 22 \\
0.70365 \pm 20 \\
0.70380 \pm 9 \\
0.70371 \pm 18 \\
0.70378 \pm 10\end{array}$ \\
\hline $\begin{array}{l}5 \times 713-2 \\
5 \times 197 \\
5 \times 341-2 \\
1 \mathrm{D} 858-3 \\
1 \mathrm{D} 872-2\end{array}$ & $\begin{array}{l}\text { Waianae (Oahu) } \\
\text { Waianae (Oahu) } \\
\text { Waianae (Oahu) } \\
\text { Kauai } \\
\text { Kauai }\end{array}$ & $\begin{array}{l}13 \\
13 \\
13 \\
14 \\
14\end{array}$ & $\begin{array}{l}21^{\circ} 24.0^{\prime} \\
21^{\circ} 21.0^{\prime} \\
21^{\circ} 20.4^{\prime} \\
21^{\circ} 59.4^{\prime} \\
21^{\circ} 59.4^{\prime}\end{array}$ & $\begin{array}{l}158^{\circ} 07.2^{\prime} \mathrm{W} \\
158^{\circ} 06.6^{\prime} \mathrm{W} \\
158^{\circ} 33.0^{\prime} \mathrm{W} \\
159^{\circ} 43.2^{\prime} \mathrm{W} \\
159^{\circ} 43.2^{\prime} \mathrm{W}\end{array}$ & $\begin{array}{l}\text { Waianae Series } \\
\text { Waianae Series } \\
\text { Waianae Series } \\
\text { Napali Formation } \\
\text { Napali Formation }\end{array}$ & $\begin{array}{l}\text { Tholeiite }(\mathrm{O}) \\
\text { Alkalic basalt }(\mathrm{O}) \\
\text { Alkalic basalt }(\mathrm{O}) \\
\text { Tholeiite }(\mathrm{O}) \\
\text { Tholeiite }(\mathrm{O})\end{array}$ & $\begin{array}{r}8.0 \\
10.9 \\
33.6 \\
4.2 \\
1.3\end{array}$ & $\begin{array}{l}422 \\
665 \\
836 \\
327 \\
324\end{array}$ & $\begin{array}{l}0.019 \\
0.016 \\
0.040 \\
0.013 \\
0.004\end{array}$ & $\begin{array}{l}0.70399 \pm 12 \\
0.70381 \pm 16 \\
0.70369 \pm 12 \\
0.70373 \pm 23 \\
0.70369 \pm 13\end{array}$ \\
\hline $\begin{array}{l}\text { 8G104 } \\
\text { 8G141-2 } \\
\text { 72HIG-51-3 } \\
\text { 72HIG-51-4 } \\
\text { 72HIG-49-1 }\end{array}$ & $\begin{array}{l}\text { Nihoa } \\
\text { Nihoa } \\
\text { Unnamed seamount } \\
\text { Unnamed seamount } \\
\text { Unnamed seamount }\end{array}$ & $\begin{array}{l}17 \\
17 \\
20 \\
20 \\
21\end{array}$ & $\begin{array}{l}23^{\circ} 03.6^{\prime} \\
23^{\circ} 03.4^{\prime} \\
23^{\circ} 15.0^{\prime} \\
23^{\circ} 15.0^{\prime} \\
23^{\circ} 20.9^{\prime}\end{array}$ & $\begin{array}{l}161^{\circ} 55.7^{\prime} \mathrm{W} \\
161^{\circ} 55.4^{\prime} \mathrm{W} \\
163^{\circ} 06.1^{\prime} \mathrm{W} \\
163^{\circ} 06.1^{\prime} \mathrm{W} \\
163^{\circ} 33.8^{\prime} \mathrm{W}\end{array}$ & $\begin{array}{l}- \\
- \\
- \\
- \\
-\end{array}$ & $\begin{array}{l}\text { Tholeiite (O) } \\
\text { Tholeiite (O) } \\
\text { Alkalic basalt (D) } \\
\text { Tholeiite (D) } \\
\text { Olivine basalt (D) }\end{array}$ & $\begin{array}{c}2.6 \\
0.4 \\
20 \\
2 \\
6\end{array}$ & $\begin{array}{l}262 \\
212 \\
880 \\
340 \\
460\end{array}$ & $\begin{array}{l}0.010 \\
0.002 \\
0.023 \\
0.006 \\
0.013\end{array}$ & $\begin{array}{l}0.70379 \pm 20 \\
0.70364 \pm 13 \\
0.70332 \pm 17 \\
0.70407 \pm 13 \\
0.70353 \pm 10\end{array}$ \\
\hline $\begin{array}{l}\text { LPP-W-20 } \\
\text { LPP-W-30 } \\
\text { 72HIG-37-1 } \\
\text { 72HIG-37-2B } \\
\text { R-1189 }\end{array}$ & $\begin{array}{l}\text { La Perouse Pinnacles } \\
\text { La Perouse Pinnacles } \\
\text { Gardner Pinnacle } \\
\text { Gardner Pinnacle } \\
\text { Midway Island }\end{array}$ & $\begin{array}{l}26 \\
26 \\
30 \\
30 \\
52\end{array}$ & $\begin{array}{l}23^{\circ} 46.0^{\prime} \\
23^{\circ} 46.0^{\prime} \\
25^{\circ} 13.3^{\prime} \\
25^{\circ} 13.3^{\prime} \\
28^{\circ} 15.5^{\prime}\end{array}$ & $\begin{array}{l}166^{\circ} 15.8^{\prime} \mathrm{W} \\
166^{\circ} 15.8^{\prime} \mathrm{W} \\
167^{\circ} 51.4^{\prime} \mathrm{W} \\
167^{\circ} 51.4^{\prime} \mathrm{W} \\
177^{\circ} 22.0^{\prime} \mathrm{W}\end{array}$ & $\begin{array}{l}- \\
- \\
- \\
- \\
-\end{array}$ & $\begin{array}{l}\text { Tholeiite (O) } \\
\text { Tholeiite (O) } \\
\text { Alkali olivine basalt (D) } \\
\text { Alkali olivine basalt (D) } \\
\text { Hawaiite (C) }\end{array}$ & $\begin{array}{c}6.4 \\
6.5 \\
9 \\
11 \\
36\end{array}$ & $\begin{array}{l}288 \\
293 \\
510 \\
550 \\
640\end{array}$ & $\begin{array}{l}0.022 \\
0.022 \\
0.018 \\
0.020 \\
0.056\end{array}$ & $\begin{array}{l}0.70393 \pm 13 \\
0.70372 \pm 8 \\
0.70324 \pm 14 \\
0.70336 \pm 10 \\
0.70379 \pm 21\end{array}$ \\
\hline
\end{tabular}




\begin{tabular}{|c|c|c|c|c|c|c|c|c|c|c|}
\hline R-1 214 & Midway Island & 52 & $28^{\circ} 15.5^{\prime}$ & $177^{\circ} 22.0^{\prime} \mathrm{W}$ & - & Mugearite (C) & 53 & 860 & 0.062 & $0.70366 \pm 8$ \\
\hline R-1625 & Midway Island & 52 & $28^{\circ} 15.5^{\prime}$ & $177^{\circ} 22.0^{\prime} \mathrm{W}$ & - & Tholeite (C) & - & - & - & $0.70375 \pm 18$ \\
\hline TSDY3-2-6 & Unnamed seamount & 63 & $29^{\circ} 49.7^{\prime}$ & $179^{\circ} 03.7^{\prime} \mathrm{W}$ & - & Alkalic basalt (D) & 13 & 420 & 0.031 & $0.70373 \pm 15$ \\
\hline \multirow[t]{2}{*}{ TSDY3-2-14 } & Unnamed seamount & 63 & $29^{\circ} 49.7^{\prime}$ & $179^{\circ} 03.7^{\prime} \mathrm{W}$ & - & Limburgite (D) & 8 & 75 & 0.107 & $\begin{array}{l}0.70437 \pm 22^{\mathrm{d}} \\
0.70460 \pm 38^{\mathrm{d}}\end{array}$ \\
\hline & & & & & & & & & & $0.70443 \pm 22$ \\
\hline TSDY3-2-16 & Unnamed seamount & 63 & $29^{\circ} 49.7^{\prime}$ & $179^{\circ} 03.7^{\prime} \mathrm{W}$ & - & Alkalic basalt (D) & 23 & 440 & 0.052 & $0.70394 \pm 9$ \\
\hline TSDY3-2-22 & Unnamed seamount & 63 & $29^{\circ} 49.7^{\prime}$ & $179^{\circ} 03.7^{\prime} \mathrm{W}$ & - & Analcime Tephrite (D) & 32 & 555 & 0.058 & $0.70351 \pm 19$ \\
\hline TSDY $3-2-30$ & Unnamed seamount & 63 & $29^{\circ} 49.7^{\prime}$ & $179^{\circ} 03.7^{\prime} \mathrm{W}$ & - & Hawaiite (D) & 95 & 640 & 0.148 & $0.70351 \pm 12^{\mathrm{d}}$ \\
\hline A7-55-25 & Daikakuji Seamount & 67 & $32^{\circ} 08.2^{\prime}$ & $172^{\circ} 15.7^{\prime} \mathrm{E}$ & - & Alkalic basalt (D) & 22 & 595 & 0.037 & $0.70386 \pm 24$ \\
\hline A7-55-29 & Daikakuji Seamount & 67 & $32^{\circ} 08.2^{\prime}$ & $172^{\circ} 15.7^{\prime} \mathrm{E}$ & - & Tholeiite (D) & 7 & 340 & 0.021 & $0.70375 \pm 14$ \\
\hline A 7-55-32 & Daikakuji Seamount & 67 & $32^{\circ} 08.2^{\prime}$ & $172^{\circ} 15.7^{\prime} \mathrm{E}$ & - & Alkali olivine basalt (D) & 8 & 665 & 0.012 & $\begin{array}{l}0.70397 \pm 15 \\
0.70393 \pm 8 \\
0.70394 \pm 9\end{array}$ \\
\hline A 7-53-11 & Yūryaku Seamount & 69 & $32^{\circ} 43.2^{\prime}$ & $172^{\circ} 12.1^{\prime} \mathrm{E}$ & - & Tholeiitic or transitional basalt (D) & 15 & 330 & 0.045 & $0.70361 \pm 15$ \\
\hline A $7-52-50$ & Kimmei Seamount & 72 & $33^{\circ} 44.0^{\prime}$ & $171^{\circ} 36.1^{\prime} \mathrm{E}$ & - & Feldspar phyric basalt (D) & 16 & 550 & 0.029 & $\begin{array}{l}0.70305 \pm 15 \\
0.70341 \pm 24 \\
0.70315 \pm 14\end{array}$ \\
\hline A $7-43-13$ & Kōkō Seamount & 74 & $34^{\circ} 46.7^{\prime}$ & $171^{\circ} 49.8^{\prime} \mathrm{E}$ & - & Alkalic basalt (D) & 12 & 380 & 0.032 & $0.70359 \pm 30$ \\
\hline A $7-43-33$ & Kōkō Seamount & 74 & $34^{\circ} 46.7^{\prime}$ & $171^{\circ} 49.8^{\prime} \mathrm{E}$ & - & Nepheline phonolite (D) & 220 & 18.4 & 11.99 & $0.70417 \pm 14^{\mathrm{d}}$ \\
\hline A $7-43-51$ & Kōkō Seamount & 74 & $34^{\circ} 46.7^{\prime}$ & $171^{\circ} 49.8^{\prime} \mathrm{E}$ & - & Tholeiite (D) & 8 & 289.4 & 0.028 & $0.70319 \pm 22$ \\
\hline \multirow[t]{2}{*}{ A $7-43-82$} & Kōkō Seamount & 74 & $34^{\circ} 46.7^{\prime}$ & $171^{\circ} 49.8^{\prime} \mathrm{E}$ & - & Alkalic basalt (D) & 28 & 980 & 0.029 & $\begin{array}{l}0.70308 \pm 14 \\
0.70307 \pm 14\end{array}$ \\
\hline & & & & & & & & & & $0.70308 \pm 10$ \\
\hline A $7-43-89$ & Kōkō Seamount & 74 & $34^{\circ} 46.7^{\prime}$ & $171^{\circ} 49.8^{\prime} \mathrm{E}$ & - & Hawaiite (D) & 30 & 217.1 & 0.014 & $0.70313 \pm 17$ \\
\hline A 7-44-5 & Kōkō Seamount & 77 & $35^{\circ} 34.9^{\prime}$ & $170^{\circ} 57.2^{\prime} \mathrm{E}$ & - & Tholeiite (D) & 10 & 365 & 0.027 & $0.70353 \pm 11$ \\
\hline A $7-44-11$ & Kōkō Seamount & 77 & $35^{\circ} 34.9^{\prime}$ & $170^{\circ} 57.2^{\prime} \mathrm{E}$ & - & Tholeiite (D) & 13 & 360 & 0.036 & $0.70367 \pm 17$ \\
\hline $430 \mathrm{~A}-4-2,110-118 \mathrm{~cm}$ & $\overline{\mathrm{O}}$ jin Seamount & 81 & $37^{\circ} 59.3^{\prime}$ & $170^{\circ} 35.9^{\prime} \mathrm{E}$ & - & Hawaiite (C) & 33 & 634.8 & 0.052 & $0.70353 \pm 12$ \\
\hline $430 \mathrm{~A}-5-1,21-27 \mathrm{~cm}$ & Ojin Seamount & 81 & $37^{\circ} 59.3^{\prime}$ & $170^{\circ} 35.9^{\prime} \mathrm{E}$ & - & Hawaiite (C) & 29 & 667.5 & 0.043 & $0.70349 \pm 7$ \\
\hline $430 \mathrm{~A}-6-3,52-63 \mathrm{~cm}$ & $\bar{O}_{j i n}$ Seamount & 81 & $37^{\circ} 59.3^{\prime}$ & $170^{\circ} 35.9^{\prime} \mathrm{E}$ & - & Hawaiite (C) & 25 & 641.3 & 0.039 & $0.70343 \pm 32$ \\
\hline $430 \mathrm{~A}-6-4,7-15 \mathrm{~cm}$ & Ojin Seamount & 81 & $37^{\circ} 59.3^{\prime}$ & $170^{\circ} 35.9^{\prime} \mathrm{E}$ & - & Hawaiite (C) & 30 & 608.8 & 0.049 & $0.70351 \pm 24$ \\
\hline $430 \mathrm{~A}-6-4,140-150 \mathrm{~cm}$ & $\vec{O}_{j i n}$ Seamount & 81 & $37^{\circ} 59.3^{\prime}$ & $170^{\circ} 35.9^{\prime} \mathrm{E}$ & - & Tholeiite (C) & 6.0 & 427.2 & 0.014 & $0.70333 \pm 28$ \\
\hline $432 \mathrm{~A}-2-3,37-43 \mathrm{~cm}$ & Nintoku Seamount & 86 & $41^{\circ} 20.0^{\prime}$ & $170^{\circ} 22.7^{\prime} \mathrm{E}$ & - & Alkalic basalt (C) & 26 & 650.2 & 0.040 & $0.70335 \pm 25$ \\
\hline $432 \mathrm{~A}-3-2,120-126 \mathrm{~cm}$ & Nin toku Seamount & 86 & $41^{\circ} 20.0^{\prime}$ & $170^{\circ} 22.7^{\prime} \mathrm{E}$ & - & Alkalic basalt (C) & 22 & 522.7 & 0.042 & $0.70334 \pm 27$ \\
\hline $432 \mathrm{~A}-5-2,57-66 \mathrm{~cm}$ & Nintoku Seamount & 86 & $41^{\circ} 20.0^{\prime}$ & $170^{\circ} 22.7^{\prime} \mathrm{E}$ & - & Alkalic basalt (C) & 21 & 490.0 & 0.043 & $0.70347 \pm 13$ \\
\hline $433 \mathrm{~A}-20-1,30-36 \mathrm{~cm}$ & Suiko Seamount & 90 & $44^{\circ} 46.6^{\prime}$ & $170^{\circ} 01.3^{\prime} \mathrm{E}$ & - & Alkalic basalt (C) & 21 & 430.5 & 0.049 & $0.70290 \pm 15$ \\
\hline $433 \mathrm{~A}-21-4,129-138 \mathrm{~cm}$ & Suiko Seamount & 90 & $44^{\circ} 46.6^{\prime}$ & $170^{\circ} 01.3^{\prime} \mathrm{E}$ & - & Alkalic basalt (C) & 16 & 379.5 & 0.042 & $0.70308 \pm 10$ \\
\hline $433 \mathrm{~B}-5-2,61-68 \mathrm{~cm}$ & Suiko Seamount & 90 & $44^{\circ} 46.6^{\prime}$ & $170^{\circ} 01.3^{\prime} \mathrm{E}$ & - & Alkalic basalt (C) & 19 & 392.1 & 0.048 & $0.70323 \pm 14$ \\
\hline $433 \mathrm{C}-4-1,25-28 \mathrm{~cm}$ & Suiko Seamount & 90 & $44^{\circ} 46.6^{\prime}$ & $170^{\circ} 01.2^{\prime} \mathrm{E}$ & - & Alkalic basalt (C) & 18 & 351.8 & 0.051 & $0.70330 \pm 31$ \\
\hline $433 \mathrm{C}-15-6,16-31 \mathrm{~cm}$ & Suiko Seamount & 90 & $44^{\circ} 46.6^{\prime}$ & $170^{\circ} 01.2^{\prime} \mathrm{E}$ & - & Tholeiite (C) & 1.6 & 305.4 & 0.005 & $0.70316 \pm 10$ \\
\hline $433 \mathrm{C}-17-1,69-82 \mathrm{~cm}$ & Suiko Seamount & 90 & $44^{\circ} 46.6^{\prime}$ & $170^{\circ} 01.2^{\prime} \mathrm{E}$ & - & Tholeiite (C) & 5.1 & 295.8 & 0.017 & $0.70326 \pm 12$ \\
\hline $433 \mathrm{C}-28-2,73-80 \mathrm{~cm}$ & Suiko Seamount & 90 & $44^{\circ} 46.6^{\prime}$ & $170^{\circ} 01.2^{\prime} \mathrm{E}$ & - & Tholeiite (C) & 6.7 & 294.0 & 0.023 & $0.70324 \pm 12$ \\
\hline $433 \mathrm{C}-29-2,94-100 \mathrm{~cm}$ & Suiko Seamount & 90 & $44^{\circ} 46.6^{\prime}$ & $170^{\circ} 01.2^{\prime} \mathrm{E}$ & - & Tholeiite (C) & 6.9 & 298.2 & 0.023 & $0.70336 \pm 16$ \\
\hline $433 \mathrm{C}-31-1,28-34 \mathrm{~cm}$ & Suiko Seamount & 90 & $44^{\circ} 46.6^{\prime}$ & $170^{\circ} 01.2^{\prime} \mathrm{E}$ & - & Tholeiite (C) & 5.2 & 327.4 & 0.016 & $0.70321 \pm 16$ \\
\hline
\end{tabular}

aFrom Barger and Jackson (1974).

eSr concentrations in italics measured by isotope dilution. Other $\mathrm{Sr}$ concentrations and all $\mathrm{Rb}$ concentrations measured by X-ray fluorescence, except for analyses by D. A. Clague made by atomic absorption. Analysts were as follows: isotope dilution, A. L. Berry and M. A. Lanphere; atomic absorption, D. A. Clague - HIG, Midway, TSDY, and A7 suites; X-ray fluorescence

HIG, Midway, TSDY, and A7 suites; J. Morris, DSDP samples; W. Doering, remainder.
$\mathrm{d} 86 \mathrm{Sr} / 88 \mathrm{Sr}$ ratios ages. Values in italics are weighted mean values for replicate measurements calculated by weighting individual measurements by the inverse of their estimated variances. Errors are estimated standard deviations of analytical precision. 


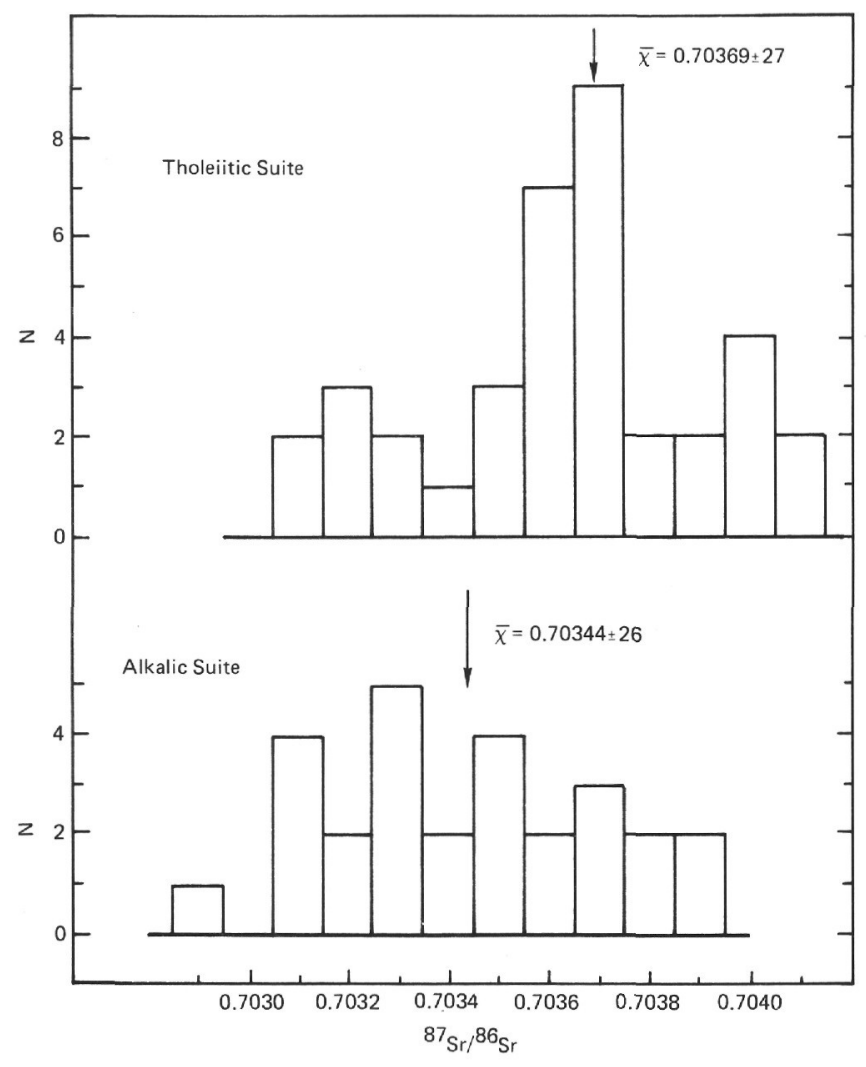

Figure 3. Histograms of the ${ }^{87} \mathrm{Sr} / 86 \mathrm{Sr}$ ratios of 37 tholeiites and 27 alkalic basalts from the Hawaiian-Emperor chain.

and alkalic basalts from the five volcanoes on the island of Hawaii indicate that the basalts originate from the same source region or closely related source regions, and that the alkalic basalts are fractionation products of tholeiitic magma. The Sr isotopic data are not compatible with this latter conclusion.

The five basalts of the nephelinic suite (Clague et al., 1975) probably have no direct relation to construction of the shield volcano (no. 63 of Bargar and Jackson, 1974) from which the samples were dredged if these nephelinic basalts were formed in a manner analogous to the Honolulu Volcanic Series on Oahu. K-Ar ages indicated that the Honolulu Series basalts were erupted from vents at least a million years after the shieldbuilding volcanism had ceased (Lanphere and Dalrymple, in press). The ${ }^{87} \mathrm{Sr} / 86 \mathrm{Sr}$ ratios of nephelinic basalts of the Honolulu Series are lower than ratios of tholeiites from the Koolau shield volcano. Relations of the five nephelinic basalts analyzed in this study are not as clear. Two of them have high $\mathrm{Rb} / \mathrm{Sr}$ ratios (Table 2), and an age correction had to be applied to the measured ${ }^{87} \mathrm{Sr} /{ }^{86} \mathrm{Sr}$ ratios. The corrections were -0.00092 and -0.00038 for TSDY $3-2-14$ and TSDY 3-2-30, respectively. Making an age correction adds additional uncertainty to the initial $\mathrm{Sr}$ composition, but this is not serious for these two samples. Since all the nephelinic basalts are from one volcano, it is not possible to characterize this suite as a group comparable to the tholeiitic and alkalic suites.
A question to ask is whether the variation in $\mathrm{Sr}$ isotopic compositions within the tholeiitic and alkalic suites represents real geological differences or merely analytical scatter. This can be tested by comparing the standard deviation of the ${ }^{87} \mathrm{Sr} /{ }^{86} \mathrm{Sr}$ ratios for each suite against the estimate of analytical precision (the pooled estimate of standard deviation for replicate measurements), using an F-test. The test results indicate that, at the 99 per cent confidence level, the variations in $\mathrm{Sr}$ isotopic composition of both suites are geological, not analytical. That is, although the source regions for the basalts may be similar in isotopic composition, the source regions for individual volcanoes and even individual flow units have variable ${ }^{87} \mathrm{Sr} / 86 \mathrm{Sr}$ ratios.

A plot of ${ }^{87} \mathrm{Sr} /{ }^{86} \mathrm{Sr}$ versus distance from Kilauea (Figure 4) shows some interesting characteristics. In general, the ${ }^{87} \mathrm{Sr} / 86 \mathrm{Sr}$ ratios are reasonably uniform in basalts along the Hawaiian Islands from Kilauea to the Hawaiian-Emperor bend, ranging only from about 0.7036 to 0.7040 . Basalts on the Emperor Seamounts north of the bend, however, have ${ }^{87} \mathrm{Sr} / 86 \mathrm{Sr}$ ratios that are significantly lower, ranging from about 0.7032 to 0.7035 . This relationship is shown diagramatically for the tholeiites in Figure 5, where an envelope has been arbitrarily drawn through the maximum and minimum ${ }^{87} \mathrm{Sr} / 86 \mathrm{Sr}$ ratios measured on samples from an individual volcano. Data from this study and from Hart (1973) and O'Nions et al. (1977) are included in Figure 5. Results from other studies are not included, because of lower analytical precision. In the main Hawaiian Islands, the envelope is irregular, reflecting both a greater density of volcanoes and significant differences in Sr isotopic composition between adjacent volcanoes. The envelope is relatively smooth from La Perouse Pinnacles northwestward along the Hawaiian-Emperor chain.

The systematic change in the isotopic composition of $\mathrm{Sr}$ in basalts northward from the Hawaiian-Emperor bend is a striking feature. About $43 \mathrm{~m} . \mathrm{y}$. ago, the direction of motion of the Pacific plate changed dramatically, and this produced the bend in the Hawaiian-Emperor chain (Dalrymple and Clague, 1976). The $\mathrm{Sr}$ isotopic data suggest that the ${ }^{87} \mathrm{Sr} / 86 \mathrm{Sr}$ ratios increased between 65 and $43 \mathrm{~m}$.y. ago, when they reached the level that remained about the same as that at present. This pattern must reflect changes in the chemistry of the source region from which the basalts were derived. The age difference between the volcanoes and the oceanic crust on which they were built offers a possible explanation. Unfortunately, there are no magnetic anomaly lineations in the vicinity of most of the Hawaiian Ridge and Emperor Seamounts. However, the synthesis of Hilde et al. (1976) and their late Mesozoic sea-floor spreading model and the reconstruction of Jackson et al. (1978) permit some speculation. The difference in age between volcano no. 63 and the underlying oceanic crust is about 80 m.y., the same difference at the Hawaiian-Emperor bend is about 70 m.y., and the difference at the island of Hawaii is about 90 m.y. This difference seems to decrease progressively along the Emperor Seamounts. For example, the difference in age at Suiko Seamount is about 30 m.y. and at Meiji Guyot is less than 20 m.y. This indicates that the Emperor Sea- 


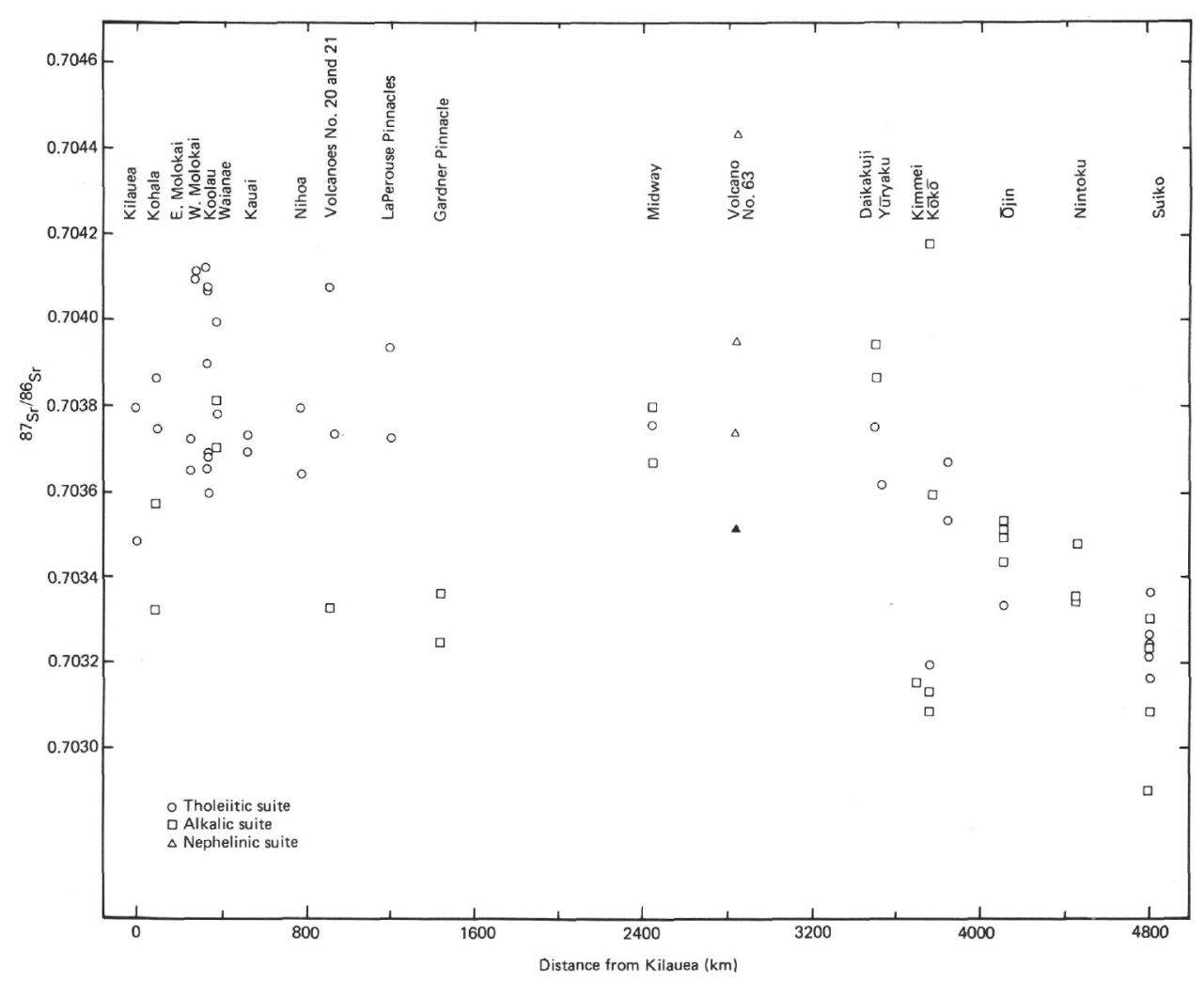

Figure 4. Plot of ${ }^{87} \mathrm{Sr} /{ }^{86} \mathrm{Sr}$ ratios as a function of distance from Kilauea Volcano measured along trend of Hawaiian Islands and Emperor Seamounts. Filled symbol indicates there are two samples at that datum.

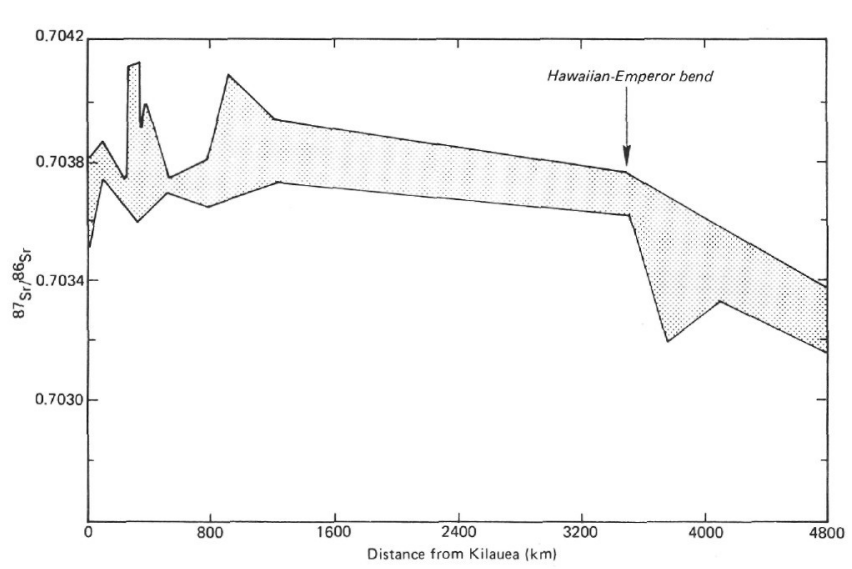

Figure 5. The range of ${ }^{87} \mathrm{Sr} / 86 \mathrm{Sr}$ ratios in tholeiites as a function of distance from Kilauea volcano. The shaded area is an envelope about minimum and maximum values measured on tholeiites from individual volcanoes on which at least two samples of tholeiitic basalt were analyzed. Data included are from this study, Hart (1973), and O'Nions et al. (1977).

mounts were formed closer to a spreading center than volcanoes on the Hawaiian Ridge, and that, within the Emperor chain, the northern volcanoes were formed closer to a spreading center then the southern volcanoes. Suiko Seamount, for example, probably was formed only about $500 \mathrm{~km}$ south of the spreading center along the Kula Ridge (Kono, this volume). The ${ }^{87} \mathrm{Sr} /{ }^{86} \mathrm{Sr}$ ratios of basalts from the Emperor Seamounts change progressively from values typical of basalts on the Hawaiian Ridge to values in the direction of mid-ocean ridge basalts (MORB), which typically have ${ }^{86} \mathrm{Sr} /{ }^{86} \mathrm{Sr}$ ratios less than 0.703 . These data imply that the $\mathrm{Sr}$ isotopic composition in basalts from volcanoes formed closer to the spreading center was, in some fashion, influenced by the age and perhaps thickness of the crust on which the volcanoes were constructed, or by the distance from the spreading ridge.

If it is assumed that the change in $\mathrm{Sr}$ isotopic composition in basalts of the Emperor Seamounts continues northward, then the predicted ${ }^{87} \mathrm{Sr} / 86 \mathrm{Sr}$ ratios of basalts erupted from a volcano located at the spreading center would be 0.7030 to 0.7032 . Sr isotopic compositions of this order have been measured for basalts from Iceland (Hart et al, 1973), a locus of oceanic island volcanism located on an active spreading center. In the Azores Islands east of the Mid-Atlantic ridge, basalts erupted farther from the ridge have higher ${ }^{87} \mathrm{Sr} /{ }^{86} \mathrm{Sr}$ ratios than basalts erupted nearer the ridge (White et al., 1975).

The locus of volcanism at the southeastern end of the Hawaiian Islands is the type example of a "hot spot." Although the hypothesis that the hot spot is the surface expression of a thermal plume (Morgan 1972a, b) has received perhaps the most attention, other ideas about the nature of the Hawaiian melting anomaly have been outlined. These include, among others, propagating fractures (Betz and Hess, 1942; Jackson and Wright, 
1970; McDougall, 1971), diapiric upwelling (Green, 1971), shear melting (Shaw, 1973), and shear melting stabilized by a downward moving plume of refractory material (gravitational anchor) (Shaw and Jackson, 1973). All of these ideas can be grouped according to whether the melting anomaly is located in the deep mantle, in the asthenosphere, or in the lithosphere.

Narrow plumes of material rising from deep in the mantle might be expected either to be chemically homogeneous or to change only very slowly, because they result from convection that should mix large volumes of mantle material and thus smooth out at least small-scale variations in composition. If this premise is correct, the composition of material in the rising plume could change gradually as a function of time, but rapid variations should not occur. However, the significant variations in Sr-isotopic composition between adjacent volcanoes, particularly in the main Hawaiian Islands, indicate that the source of the basalt magmas is heterogeneous, not homogeneous, on a short-term time scale and over rather small (intra-hot-spot) distances.

The models involving a melting anomaly in the asthenosphere do not require the region of melting to be chemically homogeneous. If the melting region is fixed relative to the mantle, then the constituents removed and erupted on the surface must be replenished by some mechanism involving lateral or vertical flow, or both, in the asthenosphere. Paleomagnetic evidence cited earlier requires the Hawaiian melting anomaly to have been relatively near its present latitude for the past $65 \mathrm{~m} . \mathrm{y}$. The significant variations in the isotopic compositions of $\mathrm{Sr}$ and $\mathrm{Pb}$ in oceanic basalts (for example, Tatsumoto, 1978) require that the oceanic mantle be heterogeneous in $\mathrm{Rb} / \mathrm{Sr}$ and $\mathrm{U} / \mathrm{Pb}$ ratios. If the melting anomaly is replenished by sampling a heterogeneous mantle, then the heterogeneities probably would carry through into the derivative basalts, though the sampling mechanism might tend to homogenize the source region.

Another potential source of magma is the Pacific plate itself as it is translated across a melting anomaly in the asthenosphere. In this case, the melting anomaly (that is, rising plume) is only an energy source that provides the heat required to produce melting in the overlying lithospheric plate. Experimental petrologic evidence for depths at which partial melting occurs to produce basaltic liquids (for example, Green, 1971) and the observations of the depth of volcanic tremor beneath Kilauea Volcano (Eaton, 1962, 1967) are consistent with magma source within the Pacific plate.

Hedge (1978) recently suggested that the basalts erupting in the Pacific Ocean basin exhibit a simple geometric pattern which indicates the presence of areas of depleted, enriched, and undepleted mantle beneath the Pacific Ocean. He suggested that many of the oceanic island basalts, including those from the main Hawaiian Islands, are derived from a widespread undepleted mantle layer having a ${ }^{87} \mathrm{Sr} / 86 \mathrm{Sr}$ ratio of about 0.7034 . This, of course, is a broadly averaged value, because the ${ }^{87} \mathrm{Sr} / 86 \mathrm{Sr}$ ratios actually measured in basalts require sizeable variations in the isotopic composition of $\mathrm{Sr}$ in the source region. The data obtained in this study suggest that the averaging of Hedge tends to obscure the detailed evolution of island chains such as the Hawaiian-Emperor volcanic chains.

Trace-element and isotopic data have been used as evidence that the mantle source region for some basalts has been depleted in LIL elements by previous partial melting processes. Tatsumoto et al., (1965) first showed that the $\mathrm{Rb} / \mathrm{Sr}$ ratios of mid-ocean ridge tholeiitic basalts were too low to generate the observed ${ }^{87} \mathrm{Sr} /{ }^{86} \mathrm{Sr}$ ratios. They concluded that the source region for the basalts had been differentiated (that is, depleted in ${ }^{87} \mathrm{Rb}$ ) at some time since the mantle was initially formed. $\mathrm{A} \mathrm{Rb} / \mathrm{Sr}$ ratio of about 0.025 in the mantle source is required in order to generate the ${ }^{87} \mathrm{Sr} /{ }^{86} \mathrm{Sr}$ ratios measured in Hawaiian basalts. On this basis, about half the basalts in this study were at least in part derived from mantle source regions that were depleted in $\mathrm{Rb}$ relative to $\mathrm{Sr}$ at some time or times in the past. The other half have undergone shallow fractionation to increase the $\mathrm{Rb} / \mathrm{Sr}$ ratios. But since the overall chemistry of the basalts is similar, it probably is safe to infer that none of the Hawaiian-Emperor basalts was derived directly from undepleted mantle material.

Numerous workers have noted a positive correlation between ${ }^{87} \mathrm{Sr} /{ }^{86} \mathrm{Sr}$ and $\mathrm{Rb} / \mathrm{Sr}$ in oceanic island basalts from several areas (Sun and Hanson, 1975; Brooks et al., 1976; Duncan and Compston, 1976; Hedge, 1978). These workers also suggested that this correlation could be interpreted as a mantle isochron which dates a major differentiation event of the mantle. The Hawaiian-Emperor data do not show this correlation (Figure 6). There is little variation between ${ }^{87} \mathrm{Sr} /{ }^{86} \mathrm{Sr}$ in the HawaiianEmperor basalts, so there is essentially no correlation between ${ }^{87} \mathrm{Sr} /{ }^{86} \mathrm{Sr}$ and $\mathrm{Rb} / \mathrm{Sr}$. The correlation coefficients for the tholeiitic and alkalic suites are -0.094 and 0.008 , respectively; the correlation coefficient for the two suites pooled is -0.163 . Even if the concept of mantle isochrons has merit, the $\mathrm{Rb}-\mathrm{Sr}$ data from the Hawaiian Islands and Emperor Seamounts do not correlate in any way which might be interpreted as showing when the source region for the basalts was established.

\section{CONCLUSIONS}

The isotopic composition of $\mathrm{Sr}$ in tholeiitic and alkalic basalts from the Hawaiian Islands and Emperor Sea-

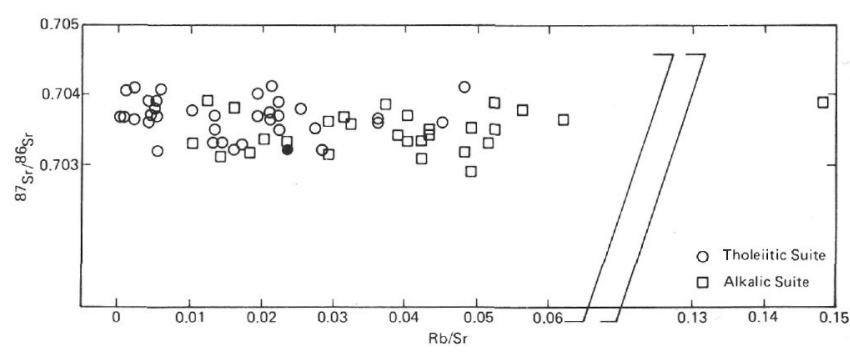

Figure 6. Plot of ${ }^{87} \mathrm{Sr} / 86 \mathrm{Sr}$ versus $\mathrm{Rb} / \mathrm{Sr}$ for basalts analyzed in this study. Samples of nephelinic suite are not included. Filled symbol indicates two samples plot at the same point. Note break in Rb/Sr scale. 
mounts is relatively uniform on a broad scale. This is consistent with derivation of the basaltic magmas from a single source region. On a more detailed scale, however, the pattern of $\mathrm{Sr}$ isotopic data is more complicated. The ${ }^{87} \mathrm{Sr} / 86 \mathrm{Sr}$ ratio in tholeiites increases systematically from $0.7032-0.7034$ at Suiko Seamount (65 m.y. ago) to $0.7036-0.7038$ at the Hawaiian-Emperor bend (43 m.y. ago). For the past 43 m.y., the ${ }^{87} \mathrm{Sr} / 86 \mathrm{Sr}$ ratio in tholeiites has remained essentially constant. This pattern of $\mathrm{Sr}$ data may be related to the distance between the position where the volcano was formed and the active spreading center (Kula Ridge). Basalts from Suiko Seamount, which formed on young oceanic crust relatively close to a spreading center, have ${ }^{87} \mathrm{Sr} /{ }^{86} \mathrm{Sr}$ ratios tending toward those of MORB, whereas basalts from volcanoes along the Hawaiian Ridge, which almost certainly formed on older oceanic crust farther from a spreading center, have ${ }^{87} \mathrm{Sr} /{ }^{86} \mathrm{Sr}$ ratios characteristic of oceanic islands.

Paleomagnetic evidence indicates that for the past 65 m.y. the Hawaiian melting anomaly (hot spot) has been near its present location. We think it likely that the melting anomaly is only a thermal energy source (Detrick and Crough, 1978) that produced melting in the overlying lithospheric plate as it moved across the hot spot. Variations in $\mathrm{Sr}$ isotopic composition within individual volcanoes and between volcanoes indicate that the source region for the basalts, in this case the lithospheric plate, was quite heterogeneous on a detailed scale.

\section{ACKNOWLEDGMENTS}

We wish to thank W.B. Friesen, S. E. Sims, and J. Saburomaru for sample preparation, A. L. Berry for $\mathrm{Sr}$ isotopic composition measurements, and A. L. Berry, W. Doering, and $\mathrm{J}$. Morris for $\mathrm{Rb}$ and $\mathrm{Sr}$ concentration measurements. We also appreciate the helpful manuscript reviews of our colleagues J. G. Arth and D. A. Swanson. D. A. Clague was supported by a NRC post-doctoral associateship with the U. S. Geological Survey and by the National Science Foundation, grant OCE 76-82515, during the course of this research.

\section{REFERENCES}

Bargar, K. E. and Jackson, E. D., 1974. Calculated volumes of individual shield volcanoes along the Hawaiian-Emperor chain, U. S. Geological Survey Jour. Res., v. 2, pp. 545-550.

Bence, A. E., 1966. The differentiation history of the earth by rubidium-strontium isotopic relationships. In Massachusetts Institute of Technology 14th Annual Progress Report for 1966 (M.I.T. Dept. Geol. Geophys.), pp. 35-78.

Betz., F., Jr. and Hess, H. H., 1942. The floor of the Pacific Ocean, Geogr. Rev., v. 32, pp. 99-116.

Brooks, C., Hart, S. R., Hofmann, A., and James, D. E., 1976. Rb-Sr mantle isochrons from oceanic regions, Earth Planet. Sci. Lett., v. 32, pp. 51-61.

Christofferson, E., 1968. The relationship of sea-floor spreading in the Pacific to the origin of the Emperor Seamounts and the Hawaiian Island chain, Trans. Amer. Geophys. Union, v. 49, p. 214.

Clague, D. A., 1974. The Hawaiian-Emperor Seamount Chain: Its origin, petrology, and implications for plate tectonics, Unpub. Ph.D. thesis, Univ. Calif. at San Diego, 319 pp.
Clague, D. A., Dalrymple, G. B., and Moberly, R., 1975. Petrography and K-Ar ages of dredged volcanic rocks from the western Hawaiian Ridge and the southern Emperor Seamount chain, Geol. Soc. America Bull., v. 86, pp. 991-998.

Dalrymple, G. B. and Clague, D. A., 1976. Age of the Hawaiian-Emperor bend, Earth Planet. Sci. Lett., v. 31, pp. 313-329.

Detrick, R. S. and Crough, S. T., 1978. Island subsidence, hot spots, and lithospheric thinning, Jour. Geophys. Res., v. 83, pp. 1236-1244.

Doering, W. P., 1968. A rapid method for measuring the $\mathrm{Rb} / \mathrm{Sr}$ ratio in silicate rocks, U. S. Geol. Survey Prof. Paper 600-C, pp. C164-C168.

Duncan, R. A. and Compston, W., 1976. Sr-isotopic evidence for an old mantle source region for French Polynesian volcanism, Geology, v. 4, pp. 728-732.

Eaton, J. P., 1962. Crustal structure and volcanism in Hawaii, Amer. Geophys. Union Geophysical Monograph 6, pp. 13-29.

1967. Evidence on the source of magma in Hawaii from earthquakes, volcanic tremor, and ground deformation, Trans. Amer. Geophys. Union, v. 48, p. 254.

Faure, G. and Hurley, P. M., 1963. The isotopic composition of strontium in oceanic and continental basalts: application to the origin of igneous rocks, Jour. Petrology, v. 4, pp. 31-50.

Gast, P. W., 1960. Limitations on the composition of the upper mantle, Jour. Geophys. Res., v. 65, pp. 1287-1297.

Green, D. H., 1971. Composition of basaltic magmas as indicators of conditions of origin: Application to oceanic volcanism, Phil. Trans. Roy. Soc. London, Ser. A, v. 268, pp. $707-725$.

Grommé, S. and Vine, F. J., 1972. Paleomagnetism of Midway Atoll lavas and northward movement of the Pacific plate, Earth Planet. Sci. Lett., v. 17, pp. 159-168.

Hamilton, E. I., 1965. Distribution of some trace elements and the isotopic composition of strontium in Hawaiian lavas, Nature, v. 206, pp. 251-253.

Hart, S. R., 1973. Submarine basalts from Kilauea rift, Hawaii: nondependence of trace element composition on extrusion depth, Earth Planet. Sci. Lett., v. 20, pp. 201-203.

Hart, S. R., Schilling, J. G., and Powell, J. L., 1973. Basalts from Iceland and along the Reykjanes Ridge: $\mathrm{Sr}$ isotope geochemistry, Nature, v. 246, pp. 104-107.

Hedge, C. E., 1966. Variations in radiogenic strontium found in volcanic rocks, Jour. Geophys. Res., v. 71, pp. 6119-6126.

1978. Strontium isotopes in basalts from the $\mathrm{Pa}$ cific Ocean Basin, Earth Planet. Sci. Lett., v. 38, pp. 88-94.

Hedge, C. E. and Walthall, F. G., 1963. Radiogenic strontium-87 as an index of geologic process, Science, v. 140, pp. 1214-1217.

Heier, K. S., Compston, W., and McDougall, I., 1965. Thorium and uranium concentrations, and the isotopic composition of strontium in the differentiated Tasmanian dolerites, Geochim. et Cosmochim. Acta, v. 29, pp. 643-659.

Hilde, T. W. C., Isezaki, N., and Wageman, J. M., 1976. Mesozoic sea-floor spreading in the North Pacific. In Sutton, G. H., Manghanani, M. H., and Moberly, R. (Ed.), The Geophysics of the Pacific Ocean Basin and its Margin: Amer. Geophys. Union Geophysical Monograph 19, pp. 205-226.

Jackson, E. D. and Wright, T. L., 1970. Xenoliths in the Honolulu Volcanic Series, Hawaii, Jour. Petrology, v. 11, pp. 405-430. 
Jackson, E. D., Koizuma, I., Kirkpatrick, R. J., Avdeiko, G., Clague, D., Dalrymple, G. B., Karpoff, A.-M., McKenzie, J., Butt, A., Ling, H. Y., Takoyama, T., Greene, H. G., and Morgan, W. J., 1978. Drilling confirms hot-spot origins, Geotimes, v. 23, pp. 23-26.

Ladd, H. S., Tracey, J. I., Jr., and Gross, M. G., 1967. Drilling on Midway Atoll, Hawaii, Science, v. 156, pp. 1088-1094.

1970. Deep drilling on Midway Atoll, U. S. Geol. Survey Prof. Paper 680-A, 22 pp.

Lanphere, M. A. and Dalrymple, G. B., in press. Age and strontium isotopic composition of the Honolulu Volcanic Series, Oahu, Hawaii, Am. Jour. Sci.

Lessing, P. and Catanzaro, E. J., 1964. $\mathrm{Sr}^{87} / \mathrm{Sr}^{86}$ ratios in Hawaiian lavas, Jour. Geophys. Res., v. 69, pp. 1599-1601.

Macdonald, G. A. and Katsura, T., 1964. Chemical composition of Hawaiian lavas, Jour. Petrology, v. 5, pp. 82-133.

McDougall, I., 1971. Volcanic island chains and sea floor spreading, Nature, v. 231, pp. 141-144.

Marshall, M., 1978. The magnetic properties of some DSDP basalts from the North Pacific and inferences for Pacific Plate Tectonics, Jour. Geophys. Res., v. 83, pp. 289-308.

Morgan, W. J., 1972a. Deep mantle convection plumes and plate motions, Am. Assoc. Petroleum Geologists Bull., v. 56, pp. 203-213.

, 1972b. Plate motions and deep mantle convection. In Shagam, R., et al. (Ed.), Studies in Earth and Space Sciences: Geol. Soc. America Mem. 132 (Hess Volume), pp. 7-22.
O’Nions, R. K., Hamilton, P. J., and Evensen, N. M., 1977. Variations in ${ }^{143} \mathrm{Nd} /{ }^{144} \mathrm{Nd}$ and ${ }^{87} \mathrm{Sr} /{ }^{86} \mathrm{Sr}$ ratios in oceanic basalts, Earth Planet. Sci. Lett., v. 34, pp. 13-22.

Powell, J. L., Faure, G., and Hurley, P. M., 1965. Strontium87 abundance in a suite of Hawaiian volcanic rocks of varying silica content, Jour. Geophys. Res., v. 70, pp. 15091513.

Powell, J. L. and DeLong, S. E., 1966. Isotopic composition of strontium in volcanic rocks from Oahu, Science, v. 153, pp. 1239-1242.

Shaw, H. R., 1973. Mantle convection and volcanic periodicity in the Pacific: Evidence from Hawaii, Geol. Soc. America Bull., v. 84, pp. 1505-1526.

Shaw, H. R. and Jackson, E. D., 1973. Linear island chains in the Pacific: result of thermal plumes or gravitational anchors? Jour. Geophys. Res., v. 78, pp. 8634-8652.

Sun, S. S. and Hanson, G. N., 1975. Evolution of the mantle: Geochemical evidence from alkali basalt, Geology, v. 3, pp. 297-302.

Tatsumoto, M., 1978, Isotopic-composition of lead in oceanic basalt and its implication to mantle evolution, Earth Planet. Sci. Lett., v. 38, pp. 63-87.

Tatsumoto, M., Hedge, C. E., and Engel, A. E. J., 1965. Potassium, rubidium, strontium, thorium, uranium, and the ratio of strontium-87 to strontium-86 in oceanic tholeiitic basalt, Science, v. 150 , pp. 886-888.

White, W. W., Hart, S. R., and Schilling, J.-G., 1975. Geochemistry of the Azores and the Mid-Atlantic Ridge: $29^{\circ} \mathrm{N}$ to $60^{\circ} \mathrm{N}$, Carnegie Inst. Washing. Yearb. 74, pp. 224-234.

Wilson, J. T., 1963a. Evidence from islands on the spreading of ocean floors, Nature, v. 197, pp. 536-538.

1963b. A possible origin of the Hawaiian Islands,

Can. Jour. Physics, v. 41, pp. 863-870. 